\title{
EXTERMINATION METHODS OF IMAGE NOISES: A REVIEW
}

B. Perumal

Department of Electronics and Communication Engineering, Kalasalingam Academy of Research and Education, Krishnankoil, Virudhunagar (Dt), (India).

E-mail:palanimet@gmail.com

ORCID: https://orcid.org/0000-0003-4408-9396

R. Sindhiya Devi

Department of Electronics and Communication Engineering, Kalasalingam Academy of

Research and Education, Krishnankoil, Virudhunagar (Dt), (India).

E-mail: sindhiyadevi14@gmail.com

ORCID: https://orcid.org/0000-0002-7529-6438

M. Pallikonda Rajasekaran

Department of Electronics and Communication Engineering, Kalasalingam Academy of

Research and Education, Krishnankoil, Virudhunagar (Dt), (India).

E-mail: mpraja80@gmail.com

ORCID: https://orcid.org/0000-0001-6942-4512

\section{Citación sugerida:}

Perumal, B., Sindhiya, R., y Pallikonda, M. (2021). Extermination methods of image noises: a review. 3C Tecnología. Glosas de innovación aplicadas a la pyme, Edición Especial, (noviembre, 2021), 243-259. https:// doi.org/10.17993/3ctecno.2021.specialissue8.243-259 


\section{ABSTRACT}

To filter an image is necessary in the preprocessing of images for the extermination of noise. Noise is an important factor that affects the information or details in the input image and so it is a need to remove it. Here, we examine a diversified amount of denoising approaches of MRI images by reviewing the positivity and weakness of every method and the well - suited method for the removal of different noises that tend to appear in the MRI images have been discussed. In this paper, we study about different noises of MRI images like Gaussian noise, Rician noise, Speckle noise, Salt and Pepper and the Poisson noise. Also we discuss about some of the basic error metrics like mean squared error, mean absolute error and the peak signal to noise ratio. Furthermore, we have presented a study about the filters like Mean filter, Median filter, Gaussian filter, Wiener filter, Anisotropic Diffusion, Lee and Frost filter, Non Local Means filter and neural network filters in order to eliminate the noises of the MRI images. The advanced technique using neural network tops the list as it follows the training approach. Each of these filters is better in some specific manner and so hybrid filters with the better features of these filters will provide greater accuracy and robustness.

\section{KEYWORDS}

Filter, Noise, positivity, Weakness, MRI Images. 


\section{INTRODUCTION}

Brain is a mind - boggling organ having its size of about only 3 pounds, it is responsible for most of the functions of our human body. Any wreckage to the brain tissue will lead to malfunction of the entire system. One such ravage is the brain tumor, which can be detected with less difficulty with the help of Magnetic Resonant Images.

\subsection{MAGNETIC RESONANT IMAGE}

Medical Resonant Imaging (MRI) is a technique which is non - flamboyant type of investigation either in the exploration or rectification of hindrances in the brain. An efficacious magnetic induction with throbs of extremely high frequency waves are used here in order to beget the embellished outlook of the organs, more precisely the flabby tissues of the human body. This is performed so as to impel the innards of human body to be interpreted easily for the croakers. To explore the organs especially brain, it is the most hyperaware technique.

MRI is more impregnable as it does not adopt emanation and it is quite the contrast of $\mathrm{x}$ - ray procedure which is time - honored and the surveying computed tomography (CT). Therefore, no deformation in the chemical reaction occurs in our tissues. We can get diversified standpoints investigated from the images of these scanning processes. Also, we can effortlessly make a distinction of the soft tissues like the gray and white matters and the cerebrospinal fluid (CSF) of the brain by means of MRI images when compared with the CT images.

To perceive the tumefaction in the brain tissues, we can utilize an extensive self - activated process and to make it possible, we should be particularly clear about every minute details of the tissue image. We can get a clear image of the tissues by exiling the discordance in the images.

\subsection{MRI NOISES}

Generally, noise is a signal that randomly appears onto an image and it sometimes affects the entire result. In the medical imaging systems, it is a necessity to obtain clear and undistorted image outputs in order to get a clear cut picture of the affected parts. There exist different 
types of noises in the medical images, especially in the MRI images (Tahir et al., 2016). The major types of noises (Kumar \& Nachamai, 2017) that could be seen in the MRI images are Gaussian noise, Rician noise, multiplicative noise and the Speckle noise. Here, we discuss about these noises that affect the quality of Magnetic Resonance Images.

\subsubsection{GAUSSIAN NOISE}

An important and a common type of noise that appears in the MRI images is the Gaussian noise whose probability density function equals the Gaussian distribution (Boyat \& Joshi, 2015). This type of noise tends to happen during the image acquisition by some natural causes like poor illumination and high temperature. It is independent of the intensity of the signal caused by thermal noise. Also it does not depend on each pixel of the image.

The Gaussian noise can be also called as the Amplifier Noise as it normally present in the area where high amplification is used rather than the weak amplification areas. This noise is additive in nature (Suryanarayana et al., 2012). It means that this noise, as it is independent of the signal, it simply adds over to the original signal or image to get the distribution. The expectation of the density function of Gaussian noise is determined by the following equation,

$$
P(g)=\frac{1}{2 \pi \sigma} e^{-(g-\mu)^{2} / 2 \sigma^{2}}
$$

where, $\mu$ denotes the mean and $\sigma 2$ shows the variance

$\mathrm{g}$ specifies the gray level of the image

We know that the Gaussian noise is white. Hence it is clear that it has a flat power spectral density and it is the reason for the auto - correlation of Gaussian noise to be zero.

\subsubsection{RICIAN NOISE}

The Rician Noise is unprejudiced that is almost similar to the Gaussian noise except the fact that the Rician noise depends mainly on the magnitude of the signal at the time of its acquisition every time, whereas the Gaussian noise is independent of the original data.

Generally spoken, Rician noise originates from the complex form of the Gaussian noise but with its original frequency domain measurements. It adheres to Rice propagation (Aja- 
Fernández, Alberola-López, \& Westin, 2008) which is also called the Rice - Nakagami distribution as the measurement of the MRI signal can be obtained from the square root of aggregation of the squares of the non - discriminating Gaussian parameters. We can also say this as the Rician noise with high SNR can be considered as the Gaussian noise. Rician probability density function can be given by the following expression,

$$
P(r)=\frac{r}{\sigma^{2}} e^{-\left(\frac{r^{2}+v^{2}}{2 \sigma^{2}}\right) I_{0}\left(\frac{r v}{\sigma^{2}}\right)}
$$

where, $v$ is the strength of direct component.

$\sigma$ shows the noise' orthogonal part.

$\mathrm{I} 0($.$) is the propositional mutated Bessel cylindrical function$

The noise intensity of the Rician noise is mainly based on the brightest tissue for every acquisition of image. In dark regions which have low intensity, the Rice distribution complies with Rayleigh propagation and in intense zones where the high intensity is available, it tends to Gaussian distribution.

\subsubsection{SPECKLE NOISE}

The Speckle Noise is of course - grained type that is commonly caused by errors during the transmission of data. It also emerges due to ecological impact on the imaging locator during picture procurement. It appears similar to the Gaussian noise with the exception that its probability density function follows gamma distribution. The probability density function of the Speckle noise can be denoted as,

$$
P(s)=\frac{s^{\alpha-1} e^{\frac{-s}{a}}}{\alpha-1 ! a^{\alpha}}
$$

where, $\mathrm{a}$ is the bright region where the gray level is less.

$a^{2} a$ is its variance.

The Speckle noise is the noise that is obtained in coherent imaging of objects and is multiplicative in nature. These types of noises are commonly seen in case of ultrasound images (Kushwaha \& Singh, 2017) but also seen in MRI images. 


\subsubsection{SALT AND PEPPER NOISE}

The Salt-and-Pepper noise is a typical impulse noise as it appears grizzly with canescent pixels. It is said to be Spike noise as it is caused by sharp and sudden disturbances in the signal and it statistically drops the original values. This is the reason for why it is otherwise known as data drop noise.

The Salt and Pepper noise is attributable to the impairment of its constituents in photographic sensors, fallacious memory locale in equipments or the conveyance in a flamboyant medium. It is consistently non - discriminatory and not mutually related to Magnetic Resonance imagery and it is randomly valued (Balamurugan, Sengottuvelan, \& Sangeetha, 2013). The density function of the Salt and Pepper noise is given by the following expression,

$$
P(s p)=\left\{\begin{array}{c}
P_{a} \text { for } s p=a \\
P_{b} \text { for } s p=b \\
\text { otherwise }
\end{array} \quad\right. \text {....(4) where, a is the bright region }
$$

with less gray level

$\mathrm{b}$ is the dark region with large gray level

$\mathrm{Pa}$ and $\mathrm{Pb}$ are the density equations of region 'a' and region 'b' respectively.

We can explain the appearance of the Salt and Pepper noise in the sense of pixels. The salt noise which are the white pixels have high frequency that comes in the range of 255200, whereas the pepper noise which are the dark pixels have low frequency that ranges from $5-0$.

\subsection{ERROR METRICS}

The efficiency and accuracy of any filter could be measured by taking the consideration of some error metrics (Nain et al., 2014). These metrics can be used in tracking out the errors. There are number of error metrics and here we discuss about some of them such as MSE, MAE and PSNR. 


\subsubsection{MSE}

MSE is the Mean Squared Error which can be otherwise referred to as the mean squared deviation. To observe the eminence of the adjudicator, the value of this error is calculated. The MSE is estimated by measuring the mean of squares of the error. When we take the square root of the MSE, we get the error or deviation estimation of the root mean square. This is what we called as Root Mean Square Error which is the RMSE or Root Mean Square Deviation which is RMSD as an alternate. Since the MSE is generally think out as the variance, the RMSE can be called as the Standard Deviation as it is derived by taking the square root of its variance.

The MSE of the filtered image with respect to the original image can be expressed as the summation of the absolute squared erratum in the image which is prorated by the multiplied values of the number of rows and columns in the image.

$$
\operatorname{MSE}=(\text { abs }(\text { squared error })) /(\mathrm{M} * \mathrm{~N})
$$

where $\mathrm{M}$ and $\mathrm{N}$ are the number of rows and columns of the image respectively.

Squared error is nothing but the absolute value of variation amongst the original and the recreated images.

\subsubsection{PSNR}

PSNR is defined as the proportionality between the original signal or image and the reconstructed signal. It is often used to figure out the eminence of the recreated image. The reconstructed image quality is said to be good, if the PSNR value computed is high.

PSNR is a quality measuring test, which is equivalent in the guesstimate of the humanoid assessment of the excellence of the renovated image. The value of PSNR can be demarcated easily without difficulty by considering the MSE. The PSNR can be mathematically articulated by considering the MSE in the following way:

$$
\text { PSNR }=10 \text { log_10 (2552 / MSE })
$$

Equation (15) represents the calculation of the PSNR from the known MSE values, when each pixel is quantized by 8 bits. The MSE and the PSNR values are related in such a way 
that when the MSE lean towards zero, then the PSNR tends to infinity. It means that if there is no error in the image, then the PSNR is infinite explaining that the renovated image is of high quality.

If the MSE gets lower in its errata, the peak ratio of the reference imagery to the noisy replica increases representing the high portrait quality with exact details.

\subsubsection{MAE}

The mean absolute error is another term that is responsible for figuring out the quality of the reconstructed image. It is the error detected by calculating the mean of the absolute errata. It is used to estimate the precognition error in its relativity to the reference image. The mean absolute error can be calculated as follows:

$$
\mathrm{MAE}=(\operatorname{sum}(\text { Reference }- \text { Reconstructed })) / \mathrm{N}
$$

where $\mathrm{N}$ is the pair of forecast and observation images. This error should be small to get good quality of images.

\section{METHODOLOGY}

De - noising is quintessential for the maneuvering of images. We can adopt this de - noising functioning as the chief task or as the supplementary of other procedures. There is an existence of several methodologies for the extermination of noise. Yet, it is still bothersome to choose a highly consistent work for a reserved implementation. Here, we discuss some methodologies such as Mean filter, Median filter, Gaussian filter, Wiener filter, Anisotropic Diffusion, Lee and Frost filter and the Non Local Means filter for the noisy imagery acquired from the MRI.

\subsection{MEAN FILTER}

Mean filter is contiguous by speculating the spatial behavior and this can be done with untroublesome implementation of smoothing images. This filter replenishes the halfway point of the kernel by taking the mean of all the other values. Hence it can be also called as the Average filter. It can do the job of a filter that is an LPF (Chandel \& Gupta, 2013), 
in which the elements of the image are summed up and then divided by the total number of elements.

The formula for this filter could be denoted as follows,

$$
f[x, y]=\frac{1}{m n} \sum_{(s, t) \in S_{x y}} g(s, t)
$$

where, Sxy is the match of counterparts in a window of size mxn centralized at $(\mathrm{x}, \mathrm{y})$.

The working of the mean filter is done by marching throughout the image completely exchanging the value of each dot with the average of neighborhood pixels including it as it is the method of smoothing which is done by lessening the measure of discrepancy of intensity amongst the neighborhood pixels.

The mean filter could be used to remove Gaussian noise and Speckle noise, whereas unsuccessful in Salt and Pepper noise. It is because, when the neighbor of the pixel bestrides a border, then the filter may introduce recently found values for the picture elements on that border which may numb the border.

\subsection{GAUSSIAN FILTER}

The corrosive Gaussian filter does not induce lofty frequency inheritance. It is deliberated to be the exemplary type of filter in time domain (Singh, 2017). It promises its favor to the extent of time on an equal footing with its favor to the extent in frequency. The cutoff may not be sharp at some pass band frequencies. This is due to the fact that this filter has its Fourier transformation as Gaussian.

The Gaussian filter has the formula as given in the expression,

$$
g(x, y)=\frac{1}{2 \pi \sigma^{2}} e^{\frac{-\left(x^{2}+y^{2}\right)}{2 \sigma^{2}}}
$$

where, $\mathrm{x}$ - level stretch from outset

$\mathrm{y}-$ erect stretch from outset

$\sigma 2$ - variance of Gaussian distribution

The kernel coefficients of the Gaussian filter depend on $\sigma$ where the larger values of $\sigma$ produce more blurring in the image. These kernels are rotationally symmetric and have no 
directional bias. Gaussian filter is a dynamic, baseband filter whose kernels are separable which results in faster computation.

The Gaussian filters remove the speckle noise which is the spike noise by smoothing the image. Also, Gaussian smoothing could be used in removing the Gaussian noise, a common sort of noise in medical MRI images.

\subsection{ANISOTROPIC DIFFUSION FILTER}

Anisotropic diffusion filter is a mechanism which reduces noise from the imagery by keeping the noteworthy parts of the image untouched. It can be also called as Perona - Malik dispersion as it is presented by Perona and Malik (1990). It is highly space - variant and is locally adaptable.

The Anisotropic Diffusion filter is based on the following equation,

$$
\partial_{t} u=\operatorname{div}\left(g\left(|\nabla u|^{2}\right) \nabla u\right)
$$

where, $|\nabla \mathrm{u}| 2$ measures the larger likelihood of locations to be edges.

The Anisotropic diffusion filter is one of the effective non - linear filters which simultaneously enhances the contrast and also reduces the noise. It depends mainly on the direction of application so that it soothes the homogeneous image regions and retains the edges of the image. Hence, it is one of the well - suited filters for the removal of Rician noise.

\subsection{WIENER FILTER}

Wiener filtering works with the term of the error of squared mean and is determined to be the unsurpassed tradeoff amongst inverse filter and the smoothing filter. It is splendiferous as an endorsement frame. The Wiener filtering provokes the yield by using the rectilinear time invariant filtering of the imagery (Kazubek, 2003). It is a refinement process of rehabilitation.

The approximated formulation for the Wiener filter in the expatriation of noise is specified by,

$$
F(x, y)=\left[\frac{1}{H(x, y)} \frac{|H(x, y)|^{2}}{|H(x, y)|^{2}+K}\right] G(x, y)
$$


where, $\mathrm{K}$ is chosen manually to obtain the best visual result.

The Wiener filter is normally a beeline filter which is considered to be stationary. It considers the frequency details instead of the spatial details and it uses the discrete Fourier transform to get the high quality replica. It uses the analytical style of processing. The noise variance can be reduced by the Wiener filter thereby making the blurred images clearly visible. The Wiener filter can be used to find the power spectra of different images and make it useful in other images by taking the average of all the data where a huge set of data exist. This gives a further smoothened imagery.

During rectification purposes, it speculates the province of decadence as well as the noise. It is the process from which we can enumerate the effectiveness of the system by considering various criteria. The image refurbishment with the Wiener filter gives excellent results as it is an outstanding restoration filter. The designing of the Wiener filter is a rectilinear process and is used in removing the Gaussian and the speckle noise, but it is not much better than the median filter.

\subsection{NON - LOCAL MEANS FILTER}

The Non - Local Means filter calculates the neutral value of all the picture elements in the given image, whose weightage is made in such a way that it finds out the level of similarity of these pixels with the intend dot (Wiest-Daesslé et al., 2008). This provides more clarity after filtering with a very little loss percentage of details in contrast to the local means algorithm.

The NL means filter formula is expressed by the following equation,

$$
u(x)=\frac{1}{c(x)} \int v(y) f(x, y) d y
$$

where, $\mathrm{u}(\mathrm{x})$ - filtrated estimate of picture at spot ' $\mathrm{x}$ '

$$
\begin{aligned}
& \mathrm{v}(\mathrm{y}) \text { - unfiltrated estimate of picture at spot ' } \mathrm{y} \text { ' } \\
& \mathrm{f}(\mathrm{x}, \mathrm{y}) \text { - weighing functionate }
\end{aligned}
$$

The level of similarity of the pixels not only depends on the homogeneity of the gray level intensity, but also the geometrical configuration in the neighborhood pixels. The main function of the NL means filter is the dismissal of Rician noise. 


\subsection{MEDIAN FILTER}

The Median filter is probably a spatial filter and is indiscriminate in nature. It is useful for the smoothing of the images. In this filter, the output is obtained from the middle value of the input exemplars in the interface, after the input sampled values are sorted.

The filter formula for the median filter is expressed as follows,

$$
v[x, y]=\operatorname{median}\{u[m, n],(m, n) \in \omega
$$

where, $\omega$ describes the vicinity established by the user, rested with the location $[\mathrm{x}, \mathrm{y}]$ of the imagery.

Hence, from the above equation, it is clear that the main principle of the median filter is to restock the value of every single picture element with the middle value of the surrounding pixels, instead of averaging the pixel values (Kazubek, 2003).

Most commonly, the kernel covers odd number of pixels and so the median is the middle value. But, if it is even, then the mean of the duo middle values can be taken as the median. Prior to the start, the voids are inlaid on every margin which makes the squinching to be presented at the borders.

The median filter is mainly used for expatriating the Salt and Pepper noise. In such type of noise, the squinched signal varies from the original and so is its mean value from the true value. Hence Salt and Pepper noise could not be reduced by conventional filters like mean or Gaussian filter successfully, but median filter is well - suited as it removes the drop - out noise effectively and preserves even the small details and edges in a better way. In case of Speckle noise, it is better than the Wiener and the mean filters. It can also be used in removing the Gaussian noise, as it is a spatial filter.

\subsection{LEE AND FROST FILTER}

The Lee filter and the Frost filter are mainly designed to remove the multiplicative noise. The Lee filter was developed by Jong Sen Lee in 1981, whereas the Frost filter was invented by Frost in the year 1982 . 
The Lee filter is mainly developed for the generative speckle pattern which utilizes localized enumeration in order to maintain the fine points. It drives in line with the variance. It means that if performs the smoothening operation for the area where the variance is low but not for high variance areas.

The Lee filter formula for the removal of noise is given by the following equation,

$$
Y_{x y}=\bar{K}+W(C-\bar{K})
$$

where, Yxy is the despeckled image

$\overline{\mathrm{K}}$ is the mean of Kernel

$\mathrm{C}$ is the central element in the kernel

$\mathrm{W}$ is the filter window

The filter window value is given by,

$$
W=\frac{\sigma_{k}^{2}}{\left(\sigma_{k}^{2}+\sigma^{2}\right)}
$$

where, $\sigma^{2}$ is the variance of the reference image

$\sigma_{\mathrm{k}}{ }^{2}$ is the variance of pixels in kernel of speckled image.

The Frost filter is a linear, convolution filter. This filter is related to the figure of variance i.e., the proportion of localized deviation to the localized mean of corrupted image. Here, the image factor decreases when we abscond from the concerned pixels while increases when near at hand.

$$
D N=\sum_{n \times n} K \alpha e^{-\alpha|t|}
$$

where, $\left(\frac{4}{n \overline{\sigma^{2}}}\right)\left(\frac{\sigma^{2}}{\overline{I^{2}}}\right)$

$$
|\mathrm{t}|=|\mathrm{X}-\mathrm{X} 0|+|\mathrm{Y}-\mathrm{Y} 0|
$$

$\mathrm{K}$ - Normalized coefficient

$\bar{I}$ - Localized mean

$\Sigma$ - Localized variance

$\bar{\sigma}$ - Image factor of deviation

$\mathrm{N}$ - Dimension of roaming kernel 
The Lee filter and the Frost filter are mainly responsible for the removal of Speckle noise (Jaybhay \& Shastri, 2015) and they are better in performance than the other filters like Wiener, mean and the median filter.

\subsection{NEURAL NETWORK FILTER}

The image denoising is recently being done by using neural network approaches. The salient point in the depiction of a neural network image filter is to link an image over another with some features that are extracted in order to form a function in a neural network. Bermudez $e t$ al. (2018) used different degrees of Gaussian noise to test their proposed method which uses a skip connection based autoencoder for denoising and proved that their method towered above the FSL SUSAN software. A combination of deep neural networks with spatio temporal denoising and bolus injection of contrast agent (CA) has been proposed by Benou et al. (2017) for reducing noise. Here, the training of data is performed by adopting a Tofts pharmacokinetic (PK) model and noise realizations. They used concatenated noisy time curves from the surrounding pixels of the frontline for further betterment. Golkov et al. (2016) recommended "q-space deep learning" approach, with model-fitting steps to get the scalar properties of voxels directly from the subsampled diffusion weighted images.

\section{RESULTS}

The error metrics MSE and MAE can be compared so as to get a clear pictorial view. The MSE and the MAE are the errors and are to be lower in each and every case (Saladi \& Prabha, 2017). With this comparative evaluation of all the filters, we get a vibrant outlook that explains the mediocre values of mean square error and the mean absolute error that are the main distinctive features of an image.

From the review of various filtering methods' positivity and weakness, the basic filters such as mean, Wiener and the Gaussian filters are well and good in the removal of speckle and Gaussian noise, but median filter is better than these by providing clear and sharp edges with details. Lee and Frost are better far from the other local filters. Rician noise could be reduced with Anisotropic diffusion and non local means filter. Comparing to the basic filters, the advanced neural network could be more comfortable in the removal of noises as it adopts the proper training of data. However, hybrid techniques are improving nowadays 
which involve the combination of two or more filters in order to improve the accuracy and robustness.

\section{CONCLUSIONS}

Good quality of images is necessary in every field that involves image processing as its part, especially in medical field. Since brain is a complex organ with soft tissues, expatriation of noise is vital. In this paper, by considering the error metrics, we find that the Gaussian noise could be reduced by using the spatial filters like mean filter, Wiener filter and Median filter. We can also use Gaussian smoothing which can considerably reduce the noise. Salt and Pepper noise can be reduced efficiently by employing median filter. The filters, anisotropic diffusion and NL means are better in removing Rician noise and the Speckle noise reduction can be effectively done by Lee and Frost filters. However, the filter based on neural network is found to be more efficient in filtering noises by mapping of noisy images with the training samples and hybrid methods are far better.

\section{REFERENCES}

Aja-Fernández, S., Alberola-López, C., \& Westin, C.-F. (2008). Noise and signal estimation in magnitude mri and rician distributed images: a LMMSE approach. IEEE Transactions On Image Processing, 17(8), 1383 - 1398. https://doi.org/10.1109/ TIP.2008.925382

Balamurugan, M., Sengottuvelan, P., \& Sangeetha, K. (2013). An empirical evaluation of salt and pepper noise removal for document images using median filter. International Journal Of Computer Applications, 82(4), 17-20. https://doi.org/10.5120/14104-2139

Benou, A., Veksler, R., Friedman, A., \& Raviv, T. R. (2017). Ensemble of expert deep neural networks for spatiotemporal denoising of contrast enhanced MRI sequences. Medical Image Analysis, 42, 145-59. https://doi.org/10.1016/j.media.2017.07.006

Bermudez, C., Plassard, A. J., Davis, T. L., Newton, A. T., Resnick, S. M., \& Landman, B. A. (2018). Learning implicit brain mri manifolds with deep learning. Proceedings Of SPIE - The International Society For Optical Engineering, 10574, pii: 105741L. https://doi.org/10.1117/12.2293515 
Boyat, A. K., \& Joshi, B. K. (2015). A review paper: noise models in digital image processing. Signal \& Image Processing: An International Fournal (SIPIf), 6(2), https://doi. org/10.5121/sipij.2015.6206

Chandel, R., \& Gupta, G. (2013). Image filtering algorithms and techniques: a review. International Fournal Of Advanced Research In Computer Science And Software Engineering, 3(10), 198202.

Golkov, V., Dosovitskiy, A., Sperl, J., Menzel, M., Gzisch, M., Sämann, P., Brox, T., \& Cremers, D. (2016). Q-space deep learning: twelve-fold shorter and modelfree diffusion mri scans. IEEE Transactions On Medical Imaging, 35(5), 1344-51. https:/ / doi.org/10.1109/TMI.2016.2551324

Jaybhay, J., \& Shastri, R. (2015). A study of speckle noise reduction filters. Signal $\&$ Image Processing: An International fournal (SIPIf), 6(3), 71-80. https://doi.org/10.5121/ sipij.2015.6306

Kazubek, M. (2003). Wavelet domain image de-noising by thresholding and wiener filter. Signal Processing Letters. IEEE, 10(11). https://doi.org/10.1109/LSP.2003.818225

Kumar, N., \& Nachamai, M. (2017). Noise removal and filtering techniques used in medical images. Oriental Fournal Of Computer Science And Technology, 10(1), 103-113. http://dx.doi.org/10.13005/ojcst/10.01.14

Kushwaha, S., \& Singh, R. K. (2017). Performance comparison of different despeckled filters for ultrasound images. Biomedical \& Pharmacology fournal, 10(2), 837-845. http:// dx.doi.org/10.13005/bpj/1175

Nain, A. K., Singhania, S., Gupta, S., \& Bhushan, B. (2014). A comparative study of mixed noise removal techniques. International fournal Of Signal Processing, Image Processing And Pattern Recognition, 7(1), 405-414. https://doi.org/10.14257/ijsip.2014.7.1.37

Perona, P., \& Malik, J. (1990). Scale-space and edge detection using anisotropic diffusion. IEEE Transactions - Pattern Analysis And Machine Intelligence, 12, 629-639. https:// ieeexplore.ieee.org/document/56205 
Saladi, S., \& Prabha, N. A. (2017). Analysis of denoising filters on mri brain images. International Fournal Of Imaging Systems And Technology, 27(3), 201-208. https://doi. org/10.1002/ima.22225

Singh, V. (2017). Comparative study of algorithms/techniques for denoising of gaussian noise. International fournal Of Advanced Research In Computer Science, 8(8), 78-82. http:/ / dx.doi.org/10.26483/ijarcs.v8i8.4614

Suryanarayana, S., Deekshatulu, B. L., Kishore, K. L., \& Kumar, Y. R. (2012). Estimation and removal of gaussian noise in digital images. International fournal Of Electronics And Communication Engineering, 5(1), 23-33.

Tahir, M., Iqbal, A., \& Khan, A. S. (2016). A review paper of various filters for noise removal in mri brain image. International Journal Of Innovative Research In Computer And Communication Engineering, 4(12), 21711-21715.

Wiest-Daesslé, N., Prima, S., Coupé, P., Morrissey, S. P., \& Barillot, G. (2008). Rician Noise Removal by Non-Local Means Filtering for Low Signal-to-Noise Ratio MRI: Applications to DT-MRI. In Metaxas D., Axel L., Fichtinger G., Székely G. (eds) Medical Image Computing and Computer-Assisted Intervention-MICCAI 2008. Lecture Notes in Computer Science, vol 5242. Springer, Berlin, Heidelberg. https://doi. org/10.1007/978-3-540-85990-1_21 